Arch. Tierz., Dummerstorf 47 (2004) 1, 47-58

Aus dem Institut für Tierzucht und Tierverhalten Mariensee der Bundesforschungsanstalt für Landwirtschaft

\title{
Milchaufnahme und Wachstumsleistung von Saugkälbern der Deutschen Rotbunten und der Deutschen Schwarzbunten im alten Typ während der Winterstallhaltung Einflüsse durch das Geschlecht und das Alter des Kalbes
}

\begin{abstract}
Title of the paper: Milk ingestion and growth performance of suckler calves born during winter stalling from German Red and White breed and from old type German Black and White breed in a cow-calf operation

On 14 calves from German Red and White breed and on 17 calves from old type German Black and White breed in a cowcalf operation measurements of body weight were done repeatedly during growth after separating the calves from the dams for $150 \mathrm{~min}$ and than again after the calves had contacted the dams for $60 \mathrm{~min}$. Measurements took place always at the same daytime. Milk ingestion of calves was assessed by way of body weight difference method. Acute body weight difference varied markedly between calves and increased with age. The fraction of positive weight difference of all measurements was between $60 \%$ and 86,2 \% (male $60 \%$ and 86,2 \%; female 67,6 \% and 80,6 $\%$ ) in DRB breed and between 69,1 \% and 89,3\% (male 72,9\% and 89,3\%; female 69,1 \% and 82,6 \%) in DSB breed. Means of all measurements showed a significant difference of acute positive body weight change and of growth rate between breeds and sexes. Mean acute positive body weight changes developed with life age and body growth with different degree in male and female calves. Growth rate of calves showed greater interand intraindividual variation from 1 to 10 days of life age. Differences in growth rate between male and female calves were more profound in DRB breed than in DSB breed.
\end{abstract}

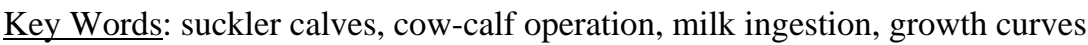

\section{Zusammenfassung}

An 14 Kälbern der Deutschen Rotbunten und 17 Kälbern der Deutschen Schwarzbunten im alten Typ der Mutterkuhherde wurden im Verlaufe der Aufzucht wiederholt Messungen der Körpermasse nach einer Separationszeit der Kälber von den Kühen von 150 min Dauer und nach einer Kontaktzeit mit den Kühen von 60 min Dauer vorgenommen. Die Messungen fanden stets in der gleichen Tageszeit statt. Aus der Körpermasse und dem Alter des Kalbes wurde die Wachstumsrate errechnet, und mit Hilfe der akuten Körpermassendifferenz (KMD) wurde die Milchaufnahme der Kälber eingeschätzt. KMD variierte im Verlaufe des Wachstums interindividuell beträchtlich und nahm mit dem Alter zu. Der Anteil der positiven KMD aller Messungen lag bei DRB zwischen $60 \%$ und 86,2 \% (m 60 \% und 86,2 \%; w 67,6 \% und 80,6 \%) und bei DSB zwischen 69,1 \% und 89,3 \% (m 72,9 \% und 89,3 \%; w 69,1 \% und 82,6 \%). An den Mittelwerten aller Messungen konnte ein signifikanter Unterschied der positiven KMD und auch der Wachstumsrate (WR) zwischen Rasse und Geschlecht nachgewiesen werden. Die mittlere positive KMD änderte sich mit zunehmendem Alter und mit der Körpermasse bei männlichen und weiblichen Kälbern in unterschiedlichem Grade. WR hatte zwischen 1 und 10 LT eine größere inter- und intraindividuelle Variation. Mittelwertunterschiede der WR zwischen männlichen und weiblichen Kälbern waren bei DRB stärker ausgeprägt als bei DSB.

Schlüsselwörter: Saugkälber, Mutterkuhhaltung, Milchaufnahme, Wachstumskurven

1. Einleitung

Das genetische Wachstumspotential der Kälber kann durch die Nahrungsverfügbarkeit und Fütterungsintensität beeinflusst werden und ist in Mutterkuhhaltungen sowohl 
von dem Verhalten und Leistungsvermögen der Kuh als auch von dem des Kalbes abhängig. Über die wechselseitige Beeinflussung der Kuh-Kalb-Paare unter verschiedenen Haltungsbedingungen liegen Untersuchungen vor, die sich auf verschiedene Rassen beziehen (ILLMANN und SPINKA, 1993; KILY-WORTHINGTON und de la PLAIN, 1983; le NEINDRE et al., 1979; le NEINDRE, 1982; LIDFORS, 1994; SPINKA und ILLMANN, 1992). Allgemein wurde angenommen, dass die Saugraten direkt proportional dem Milchtransfer sind (DAY et al., 1987; MARTIN, 1984; ODDE et al., 1985). Mit dem Alter der Kälber änderten sich die Zeitanteile der Komponenten (Vorbereitung, Saugphase, Nachsaugen) eines Saugaktes. Die eigentliche Saugphase des Saugaktes, in welcher der Milchtransfer erfolgt, soll bei allen Altersklassen der Kälber auf etwa 1,5 bis 6 min beschränkt sein und ziemlich konstant bleiben (LIDFORS, 1994; ILLMANN und SPINKA, 1993). In empirischen Untersuchungen konnten keine positiven signifikanten Beziehungen zwischen Elementen der mütterlichen Fürsorge und der Körpermasse und Wachstumsrate der Nachkommen aufgezeigt werden (MENDL und PAUL, 1989). Effekte durch das genetische Material und durch die Umgebungsbedingungen sowie typmäßige Besonderheiten wären zu erwarten, sind jedoch bisher wenig untersucht worden.

Zwischen Milchleistung und -qualität von Fleischrindkühen verschiedener Rassen und der Wachstumsleistung der Kälber konnten Korrelationen unterschiedlichen Grades festgestellt und unterschiedliche Anteile der Varianz der Absetzgewichte der Kälber erklärt werden (Übersicht bei SCHOLZ et al., 2001). Daraus geht hervor, dass weitere Faktoren einen stärkeren Einfluss auf die vorher genannten Zusammenhänge haben können. Detaillierte Angaben zur Futteraufnahme, zur Wachstumscharakteristik der Saugkälber und zu den Faktoren, die diese beeinflussen können, sind im allgemeinen spärlich. Bei einem Pilotprojekt zur Haltung von Deutschen Schwarzbunten (DSB) im alten Typ gemeinsam mit Deutschen Rotbunten (DRB) in einer Mutterkuhherde als Genreserve wurde die vorliegende Untersuchung vorgenommen und mit Hilfe der Körpermassendifferenzmethode die Futteraufnahme und der Wachstumsverlauf der Saugkälber bis zum Alter von 120 Lebenstagen (LT) charakterisiert. Von Interesse waren folgende Fragen: (1) Welchen Einfluss hat das Geschlecht des Kalbes auf die aufgenommene Milchmenge (Futtermenge)? (2) Wie ändert sich die bei dem Saugakt aufgenommene Milchmenge mit dem Alter des Kalbes? (3) Lassen sich bei den Rassenvertretern Besonderheiten der Nahrungsaufnahme nachweisen?

\section{$2 . \quad$ Material und Methoden}

\section{$2.1 \quad$ Tiere, Haltungsbedingungen}

Die Untersuchungen wurden während der Winterstallhaltungsperiode von Oktober bis April 1994/1995 vorgenommen. Die Tiere (Tab. 1) befanden sich in einem Mehrzweckgebäude, welches für die Haltung der Rinder in Laufboxen hergerichtet worden war. Die Fütterung der Kühe (Mais-, Grassilage, Heu) erfolgte zweimal täglich (07.00 bis 08.00 Uhr und 14.00 bis 15.00 Uhr) von einem zentral gelegenen Futtergang aus, der von sämtlichen Tieren der Herde zu erreichen war. Die Wasserversorgung war durch Selbsttränken und die Mineralstoffversorgung durch Leckschalen gewährleistet. Abkalbungen ereigneten sich kontinuierlich, gehäuft jedoch von Oktober bis Januar. Während der Kalbung befanden sich die Tiere in Einzelboxen mit Stroheinstreu, in welchen sie 3 bis 5 Tage verblieben. Dann kamen sie in eine Laufbox für 25 Kuh- 
Kalb-Paare, in welcher sich Kühe mit den jüngeren Saugkälbern befanden. Je nach Bedarf wurden Mutter-Kalb-Paare mit älteren Saugkälbern in eine zweite Gruppenbox mit Spaltenboden und Liegeflächen umgestallt. An beiden Gruppenboxen befand sich jeweils ein durch Gatter abgetrennter und mit Stroh eingestreuter Liegebereich für Kälber, in welchem gesondert Festfutter (Kälberfutter) und Heu angeboten werden konnten. Im Kälberliegebereich wurden die Kälber täglich für etwa 150 Minuten zwischen 12.00 und 14.30 Uhr an einem Halsgurt, den die Tiere ständig trugen, fixiert, damit sie sich an Manipulationen gewöhnen konnten. Die Separation der Kälber von den Muttertieren für eine konstante Zeitperiode und zur gleichen Tageszeit wurde für Untersuchungen über das Milch- und Futteraufnahmevermögen der Saugkälber genutzt.

Tabelle 1

Übersicht zum Tiermaterial und zu den Untersuchungen (Characteristics of the calves and number of measurements)

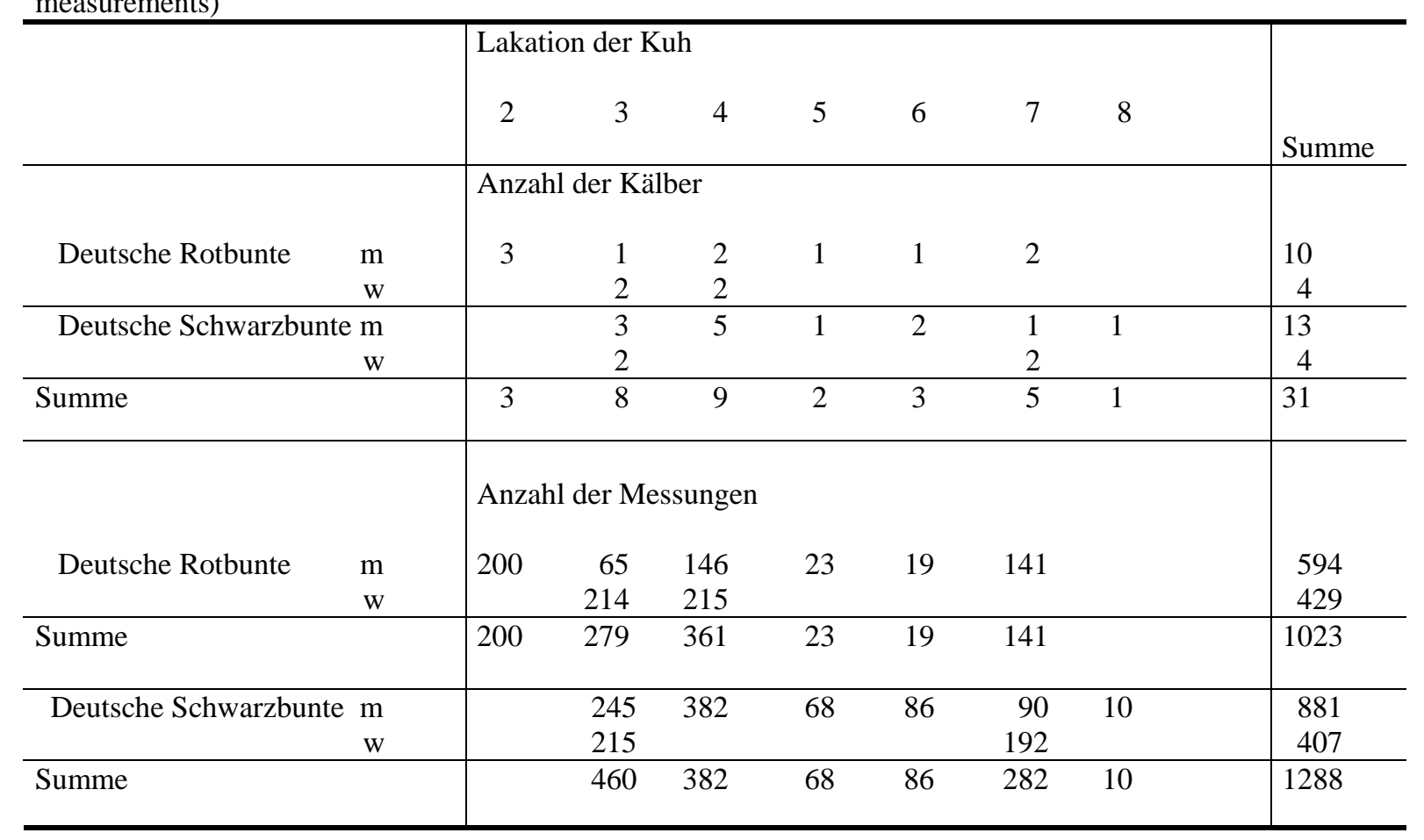

\section{$2.2 \quad$ Untersuchungsablauf, Messungen}

Die Nahrungsaufnahme der Kälber ist indirekt mit Hilfe der Körpermassendifferenzmethode festgestellt worden. Die Tiere verließen den Kälberliegebereich nach der gewohnten täglichen Separation von den Muttertieren für etwa 150 min über eine fahrbare Waage, so dass die Körpermasse vor dem Kontakt mit den Muttertieren (KM1) festgestellt werden konnte. Nach dem Kontakt mit den Muttertieren für eine Zeit von 60 min wurden sämtliche Kälber gleichzeitig wieder in den Kälberliegebereich verbracht, und die Wägung wurde noch einmal wiederholt (Körpermasse der Kälber nach dem Kontakt mit den Kühen, KM2). Die Kälber gewöhnten sich nach ein bis zwei Messungen an die Prozedur und verhielten sich bemerkenswert kooperativ, so dass die Wägungen innerhalb von 15 bis 30 min bewältigt werden konnten. Die Waage hatte einen Messbereich von $1 \mathrm{~kg}$ bis $400 \mathrm{~kg}$ und eine Skaleneinteilung von 100 g. Die akute positive Körpermassedifferenz (KM2-KM1, als KMD bezeichnet) ist für die Einschätzung der Futteraufnahme (positive KMD) genutzt worden. Als Grenz- 
wert ist die KMD 0,1 kg festgelegt worden. Orientierende Einschätzungen über Körperausscheidungen (Kot und/oder Harn) waren mit Hilfe der KMD $<0,1$ kg möglich. Geburtsgewicht, KM1 und Lebensalter der Kälber wurden für die Berechnung der Wachstumsrate (WR) genutzt (KM1-Geburtsgewicht dividiert durch das Alter in Tagen).

\subsection{Bearbeitung der Ergebnisse, statistische Methoden}

Für jedes Tier ist die mittlere WR sowie die positive und negative KMD während des Untersuchungszeitraumes und der Anteil der positiven KMD in Prozent an der gesamten Untersuchungsanzahl berechnet worden. Die Häufigkeit der KMD ist in Altersbereichen von 20 Lebenstagen bei den Rassenvertretern und Geschlechtern dargestellt worden. Bei der Prüfung der Effekte durch Rasse und Geschlecht des Kalbes auf KMD wurden Alter und Körpermasse des Kalbes als Covariate berücksichtigt. WR und KMD männlicher und weiblicher Saugkälber der beiden Rassen sind außerdem in Altersbereichen von $10 \mathrm{~d}$ während der Aufzucht geprüft worden. Der Einfluss des Geburtsgewichtes auf die KM und WR der Kälber in den Altersbereichen ist untersucht worden. Die Bearbeitung der Ergebnisse wurde mit Systat sowie Sigma Plot von SPSS Science Software vorgenommen (ANOVA und ANOVA for repeated measures, Korrelations- und Regressionsrechnung). Die Irrtumswahrscheinlichkeiten (Bonferroni adjusted probability) sind in den Tabellen und Abbildungen angegeben und, wenn nicht ausdrücklich erwähnt, mit $5 \%$ angenommen worden.

\section{3. $\quad$ Ergebnisse}

3.1 Akute Körpermassendifferenz der Saugkälber

KMD wies im Verlaufe des Wachstums zwischen den Kälbern und auch bei einzelnen Kälbern eine größere Variation auf (Abb. 1 bis 3). Die Abbildung 1 zeigt die KMD eines männlichen Kalbes mit häufiger positiver KMD (89,32 \% der Messungen) und eines solchen mit weniger häufiger positiver KMD (60,0 \%) an den Untersuchungstagen und die Wachstumskurven, an denen die unterschiedlichen WR in den ersten Lebenstagen und Perioden der Wachstumsbeschleunigung und -verzögerung auffällig sind. Die symmetrischen Dichteplots (Abb. 2) ließen die häufiger größere positive KMD männlicher Kälber insbesondere in den ersten Lebenstagen aber auch an den späteren Untersuchungspunkten erkennen. In den Altersbereichen von 61 - 80 LT (p 0,004), 81 - 100 LT (p 0,045) und 101 - 120 LT (p 0,000) war KMD männlicher Kälber der DRB und in dem Altersbereich $81-100$ LT (p 0,012) bei jenen der DSB signifikant größer als KMD weiblicher Kälber (Abb. 2). Der Anteil der positiven KMD an der gesamten Anzahl der Messungen lag in den geprüften Altersbereichen von 20 LT bei DRB zwischen 65,12 \% und 83,33 \% und bei DSB zwischen 73,08 \% und 91,27 \%. Die für die einzelnen Kälber berechneten prozentualen Anteile der positiven KMD aller Messungen pro Tier lagen bei DRB zwischen 60,0 \% und 86,15 \% (m 60 $\%$ und 86,2 \%; w 67,6 \% und 80,6 \%) und bei DSB zwischen 69,05 \% und 89,32 \% (m $72,9 \%$ und 89,3 \%; w 69,1 \% und 82,6 \%) und unterschieden sich zwischen den Rassen und Geschlechtern im Mittelwert nicht sicher. 

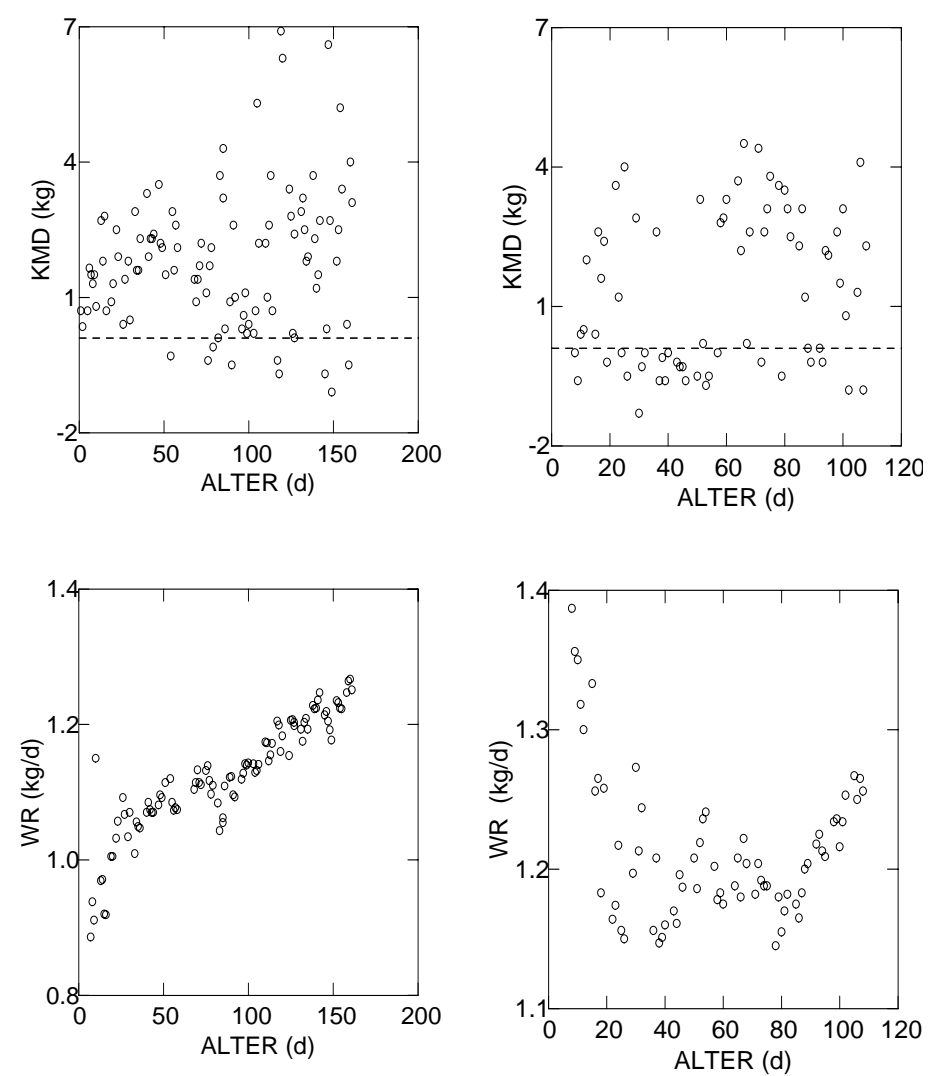

Abb. 1: Akute Körpermassendifferenz (KMD, oberer Teil) und Wachstumsrate (WR, unterer Teil) eines männlichen Kalbes (DSB, linker Teil) mit 89,32 \% positiver KMD und eines männlichen Kalbes (DRB, rechter Teil) mit 60,0 \% positiver KMD, Einzelwerte (Acute body weight difference and growth rate of a male calf from DSB breed (left) with 89,32 \% and that of a male calf from DRB breed (right) with 60,0 \% positive weight changes at testing, single values)

An den Mittelwerten aller Messungen konnte ein signifikanter Unterschied der positiven KMD zwischen Rasse und Geschlecht nachgewiesen werden (Tab. 2). Einflüsse durch die KM und durch das Alter der Saugkälber als Covariate auf KMD waren signifikant. Zwischen positiver KMD und Körpermasse sowie Alter der Kälber (männliche Kälber N 437; r = 0,21; p 0,000; $r=0,257$; p 0,000; weibliche Kälber N 322; $r$ = 0,303; p 0,000; $r=0,293$; p 0,000) ließen sich bei DRB sichere Korrelationen nachweisen. Bei DSB konnten sichere Korrelationen zwischen diesen Variablen nur bei männlichen Tieren gefunden werden ( $\mathrm{N} 710 ; \mathrm{r}=0,22 ; \mathrm{p} 0,000 ; \mathrm{r}=0,239$; $\mathrm{p}$ 0,000). Die mittleren negativen KMD lagen zwischen -0,309 kg und -0,376 kg und unterschieden sich zwischen den Kälbergruppen und den Altersbereichen nicht sicher. Effekte durch KM und Alter der Kälber auf die negative KMD waren nicht nachzuweisen. Der Anteil der negativen KMD an der gesamten Anzahl der Messungen betrug bei männlichen und weiblichen Saugkälbern der DRB $26 \%$ und 24,7 \% und bei den Kälbern der DSB 19,4 \% und 21,9 \%. 

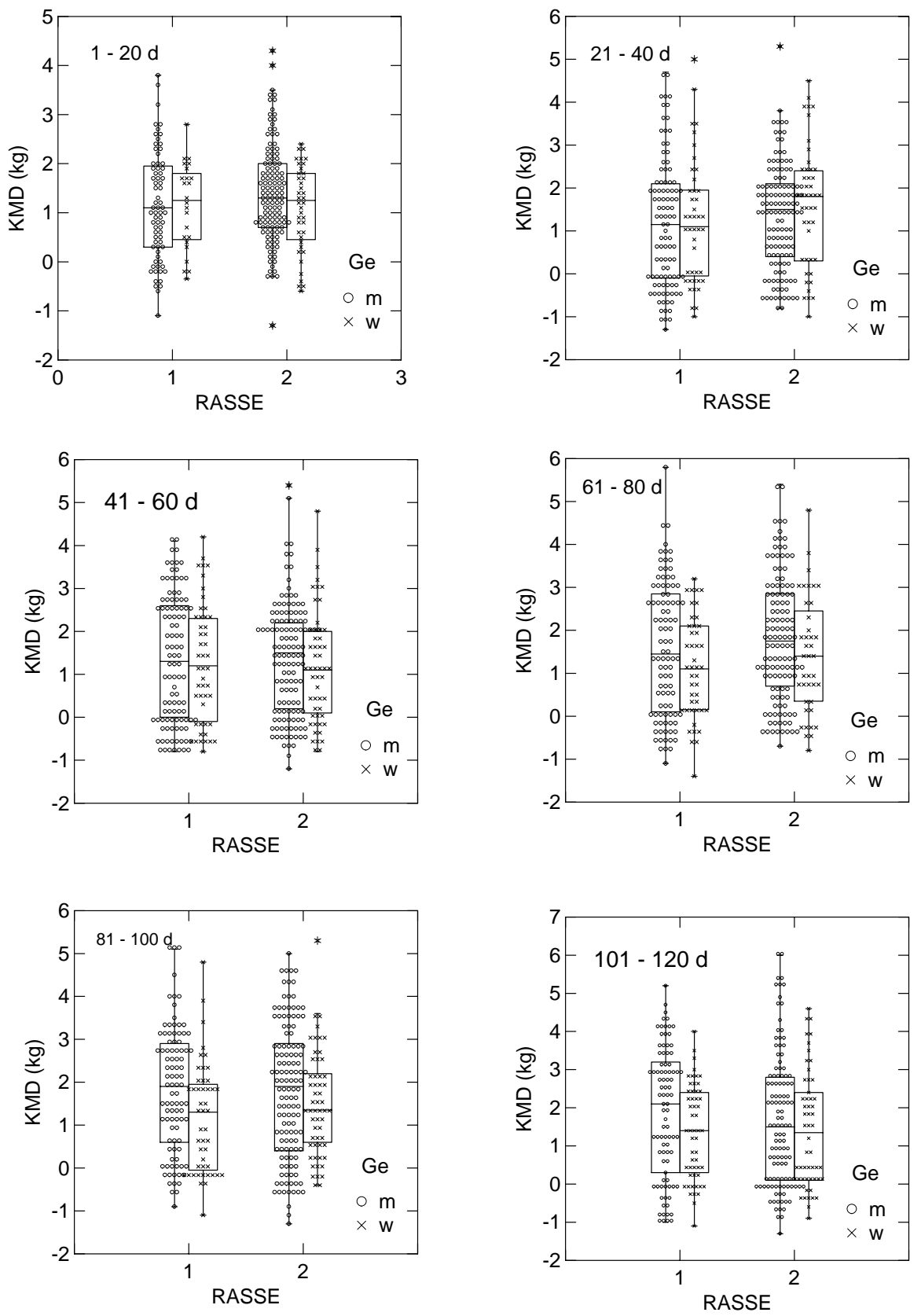

Abb. 2: Verteilung der akuten Körpermassedifferenz (KMD) in Altersperioden von 20 Lebenstagen bei männlichen und weiblichen Saugkälbern, Rasse 1: DRB; Rasse 2: DSB, Box and Whisker Plots kombiniert mit Symmetric Dot Density Plots (Distribution of acute weight changes in male and female suckler calves within life age periods of 20 days, breed 1: DRB; breed 2: DSB)

Die mittlere positive KMD wurde mit zunehmendem Alter und mit der Körpermasse der Kälber signifikant größer (Abb. 3). Dies erfolgte bei männlichen und weiblichen Kälbern in den untersuchten Alters- und Körpermassebereichen in unterschiedlichem Grade. Sicher unterschieden sich die Mittelwerte der positiven KMD der DRB im Alter von $51-60 \mathrm{LT}, 61-70 \mathrm{LT}, 101-110 \mathrm{LT}$ und $111-120 \mathrm{LT}$ und mit einer Körpermasse von $110-120 \mathrm{~kg}, 130-140 \mathrm{~kg}, 150-160 \mathrm{~kg}$ und der DSB im Alter von 61 - $70 \mathrm{LT}, 81$ - $90 \mathrm{LT}$ und 131 - $140 \mathrm{LT}$ und mit einer Körpermasse von 110 $120 \mathrm{~kg}$ sowie 140 - $150 \mathrm{~kg}$ (Abb. 3). Große mittlere KMD waren bei weiblichen Kälbern zwischen 21 und 50 LT und bei männlichen Kälbern zwischen 21 und 50 LT (DSB) sowie 21 und 70 LT (DRB) festzustellen. 

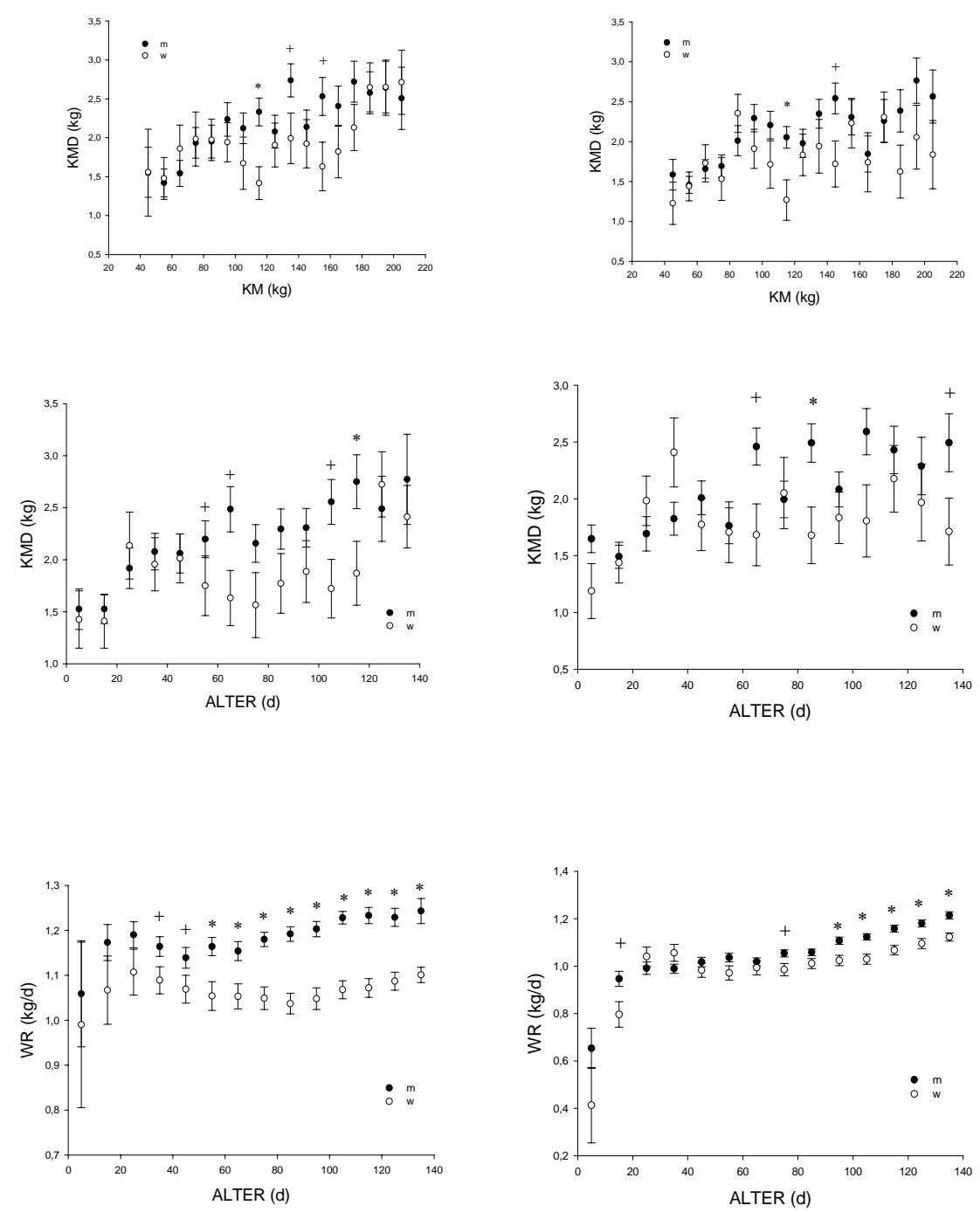

Abb. 3: Positive KMD und Körpermassebereiche (oberer Teil) sowie positive KMD (mittlerer Teil) und Wachstumsrate (unterer Teil) männlicher und weiblicher Saugkälber der DRB (linker Teil) und der DSB (rechter Teil) in Altersbereichen von 10 Lebenstagen, LSM and SEM, Mittelwertunterschiede: $+\mathrm{p}<0,05$; * $\mathrm{p}<0,01$ (Acute positive body weight changes at testing and body weight (top) and acute positive body weight changes at testing (middle) and growth rate (bottom) of male and female suckler calves from DRB breed and DSB breed within life age periods of 10 days)

\section{2}

\section{Wachstumsrate, Körpermasse}

Die WR war als Mittelwert aller Messungen bei männlichen Saugkälbern signifikant größer als bei weiblichen (Tab. 2). Der Einfluss der Rasse und eine Interaktion von Rasse und Geschlecht waren signifikant. Es ließen sich sichere Effekte durch das Alter und durch die KM der Kälber auf WR nachweisen. WR wies in dem Altersbereich von 1 - 10 LT bei einzelnen Kälbern (Abb. 1) und zwischen den Kälbern eine größere Variation als in der übrigen Zeit auf (Abb. 3) und änderte sich individuell in unterschiedlichem Grade während der Wachstumsperiode. Bei männlichen und weiblichen Kälbern vergrößerte sich die mittlere WR bis $30 \mathrm{LT}$. Unterschiede der WR männlicher und weiblicher Kälber waren bei DRB stärker ausgeprägt als bei DSB (Abb. 3). Die Körpermasse männlicher Kälber war nach der Geburt größer als die weiblicher (DRB: 46,8 $\pm 1,43 \mathrm{~kg}$ und $44 \pm 2,25 \mathrm{~kg}$; DSB: 46,18 $\pm 1,57 \mathrm{~kg}$ und 44,5 $\pm 2,6 \mathrm{~kg}$, LSM und SEM).Diese Unterschiede waren nicht signifikant. Die Körpermasse männ- 
licher Kälber vergrößerte sich insbesondere ab etwa 50 LT stärker als die weiblicher (Abb. 4). Das Geburtsgewicht hatte auf die KM der Kälber in den untersuchten Altersbereichen einen hochsignifikanten Einfluss. Mit WR hatte es bei DRB zwischen 21 und 60 LT eine schwache und zwischen 71 und 120 LT wieder eine stärkere Korrelation. Bei Kälbern der DSB war die Korrelation zwischen Geburtsgewicht und WR bis zum Alter von 30 LT signifikant negativ und in den folgenden Altersperioden nicht mehr sicher.

Tabelle 2

Wachstumsrate und akute positive und negative Körpermassedifferenz (KMD) der Saugkälber, LSM und SEM, Anzahl der Messungen in Klammern (Growth rate and acute positive and negative body weight change of suckler calves at testing, LSM and SEM, number of testings in parentheses)

\begin{tabular}{lcccc}
\hline & \multicolumn{2}{c}{ Deutsche Rotbunte } & \multicolumn{2}{c}{ Deutsche Schwarzbunte } \\
& männlich & weiblich & männlich & weiblich \\
\hline Wachstumsrate & $1,18(591)$ & $1,086(428)$ & $1,041(881)$ & $1,025(410)$ \\
$(\mathrm{kg} / \mathrm{d})$ & 0,009 & 0,011 & 0,007 & 0,011 \\
positive KMD & $2,201(437)$ & $2,149(322)$ & $2,066(710)$ & $1,833(320)$ \\
$(\mathrm{kg})$ & 0,059 & 0,069 & 0,046 & 0,069 \\
negative KMD & $-0,364(154)$ & $-0,376(106)$ & $-0,332(171)$ & $-0,309(90)$ \\
$(\mathrm{kg})$ & 0,029 & 0,035 & 0,028 & 0,038 \\
\hline
\end{tabular}
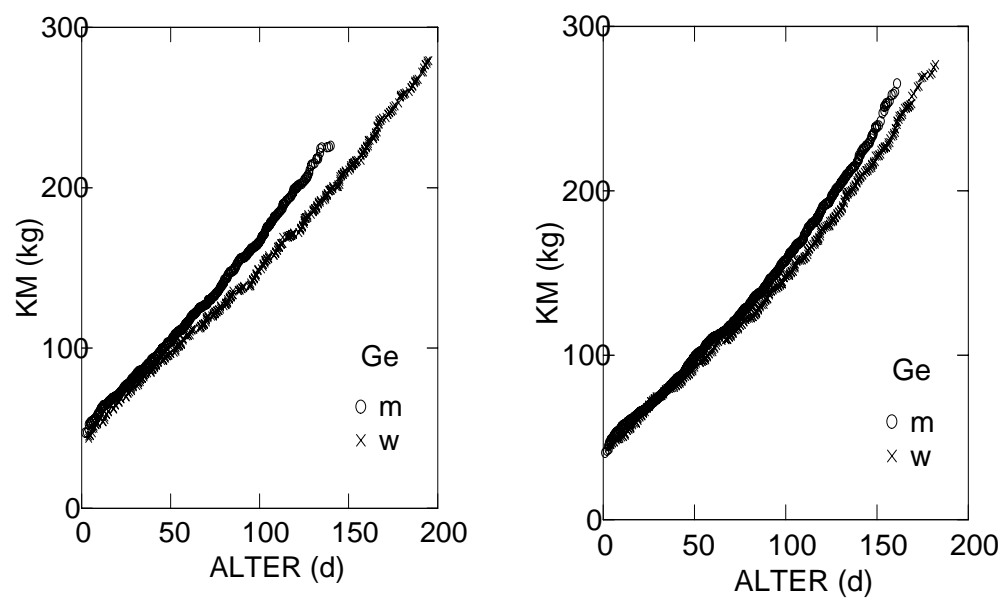

Abb. 4: Quantil-Quantil Plots des Alters und der Körpermasse männlicher und weiblicher Saugkälber der DRB (linker Teil) und der DSB (rechter Teil) (Q-Q Plots of life age and body weight of male and female suckler calves)

\subsection{Korrelationen zwischen Wachstumsrate und akuter Körpermassedifferenz}

Der prozentuale Anteil positiver KMD (y) hatte eine positive lineare Beziehung zur mittleren KMD (r 0,625; p 0,000) und eine negative lineare Beziehung zum Variationskoeffizienten der KMD ( $r=-0,892 ;$ p 0,000). Kälber, die beständiger Nahrung aufnahmen, nahmen auch größere Mengen auf.

\section{Diskussion}

Die Befunde über die Variation der von den Kälbern bei einem Saugakt aufgenommenen Milchmenge und der Körperwachstumsverläufe (Abb. 1 bis 4) ergänzten die Ergebnisse anderer Untersuchungen. Nachteile der indirekten Feststellung der von den Jungtieren aufgenommenen Milchmenge mit der Körpermassendifferenzmethode, die 
sich dann ergeben, wenn Harn und/oder Kot abgegeben und nicht gemessen worden sind, die aufgenommene Milchmenge während des periodischen kontrollierten $\mathrm{Zu}-$ ganges geringer als die Milchaufnahme während einer ungestörten Situation ist, und wenn ausschließlich oder zusätzlich zur Milch Wasser und/oder Futtermittel aufgenommen worden sind (OFTEDAL (1984), wurden bei dem gewählten Vorgehen dadurch eingeschränkt, dass sich die Tiere an die Messungen gewöhnen konnten und dass eine sehr große Anzahl von Messungen pro Tier im Verlaufe des Wachstums vorgenommen werden konnte. Die von den Kälbern während des kontrollierten Kontaktes mit der Kuh aufgenommenen Festfuttermengen haben bei älteren Kälbern eine zunehmend größere Rolle gespielt, bei jüngeren Kälbern werden sie, wenn die kurze Kontaktzeit von 60 min berücksichtigt wird, von untergeordneter Bedeutung gewesen sein. Die Wasseraufnahme der Saugkälber während der Kontaktzeit kann bei älteren Tieren nicht gänzlich ausgeschlossen werden. Der größere Anteil positiver KMD bei DSB und Mittelwertunterschiede der KMD und WR zwischen DRB und DSB sind wegen der geringen Tierzahlen vorsichtig zu bewerten.

Die Häufigkeit der positiven KMD von etwa 60,0 \% bis 89,32 \% der Messungen im Verlaufe der Aufzucht und große individuelle Unterschiede des Saugverhaltens der Kälber hinsichtlich Frequenz und Dauer (DAY et al., 1987 ; DREWEY et al., 1959; le NEINDRE et al. 1979; HOPPE, 1990; LIDFORS, 1994; LOTTMANN, 1995; ODDE et al., 1985; SPINKA und ILLMANN, 1992) sind unter dem Gesichtspunkt von Entwicklungsqualität und Alter der Kälber, der Qualität des maternalen Investment, der Herdenzusammensetzung und der Haltungsbedingungen einzuschätzen. Jüngere Kälber suchten meistens sofort den Kontakt zu den Kühen, während ältere Kälber ihre Aufmerksamkeit vermehrt auf die an dem Wägevorgang beteiligten Personen und Ereignisse konzentrierten und erst später sich der Nahrungsaufnahme zuwandten. Die durch die Nahrungsbeschaffung beeinflussten Aktivitäts- und Ruheperioden und auch die Herzfrequenz der Saugkälber wiesen bei Winterstallhaltung eine infradiane Periodik auf (STEINHARDT und THIELSCHER, 2001). Bei Weidehaltung war die Rhythmik der Aktivitäts- und Ruheperioden im Tagesverlauf synchronisiert mit jenen der Kühe, und jüngere Saugkälber wiesen in Abhängigkeit von der Nahrungsverfügbarkeit längere tägliche Gesamtzeiten der Ruhe auf als ältere (STEINHARDT, 2003). Die Beziehung zwischen der Häufigkeit der positiven KMD als Prozentsatz der Messungen und dem Mittelwert sowie dem Variationskoeffizienten der KMD und auch diejenige zwischen Körpermasse der Kälber und positiver KMD deuteten darauf hin, dass einige Kälber beständiger größere Nahrungsmengen aufnahmen als andere. Dies bestätigt die Befunde anderer Untersuchungen (SCHOLZ et al., 2001; 2002). An der gegenüber weiblichen Tieren größeren positiven KMD und WR männlicher Kälber in den ersten Lebenswochen (Abb. 3) kann eine bessere Vitalität und größere Aktivität dieser Tiere beteiligt gewesen sein. In diesem Sinne ist die Korrelation des Geburtsgewichtes mit der Körpermasse der Kälber in späteren Altersperioden einzuschätzen. Einflüsse der Entwicklungsqualität der Kälber auf die Aktivitätsperiodik und Wachstumsleistung sind bei mutterlos aufgezogenen Kälbern beschrieben worden (STEINHARDT und THIELSCHER, 2002; STEINHARDT, 2003) und können auch bei Saugkälbern von Bedeutung sein.

Größere positive KMD zwischen 20 und 40 LT bei weiblichen Kälbern insbesondere solchen der DSB (Abb. 3) sind in Verbindung mit der Feststellung zu sehen, dass weibliche Tiere einen engeren Kontakt mit den Muttertieren als männliche aufwiesen 
(LIDFORS und JENSEN, 1988; VEISSIER et al., 1990), was besonders bei der Adaptation der Nahrungssuche und -aufnahme vorteilhaft sein kann (LAZO, 1994; VEISSIER et al., 1997). Größere positive KMD männlicher Kälber im Alter von 40 bis 70 LT unter Winterstallhaltungsbedingungen (Abb. 2 und 3, Tab. 2) stehen zum Teil mit dem Fremdsaugen der Kälber in Verbindung, das mit einem Anteil von 20 bis $28 \%$ bei DSB im alten Typ oder sogar mit einem solchen von $35 \%$ der beobachteten Saugakte bei einer gemischten Herde aus DRB und DSB im alten Typ festgestellt worden war. Bei Stallhaltung wurde das Fremdsaugen der Kälber, das auch bei Weidehaltung beobachtet worden ist, anscheinend begünstigt. Vor allem ältere Kälber saugten in der Hauptphase eines Saugaktes, in welcher der Milchfluss maximal angeregt war, bei fremden Kühen. Die mittlere Dauer des Fremdsaugens lag bei 4 bis 5 min. Situationen wie die Fütterung am Gatter bei eng aneinander stehenden Kühen oder solche mit Konflikten, die sich aus der Rangordnung ergaben, in denen die Aufmerksamkeit nicht ausreichend auf das saugende Kalb gerichtet war, oder auch typmäßige Besonderheiten der Kühe wurden von den fremd saugenden Kälbern genutzt, um der Kontrolle durch das fremde Muttertier zu entgehen.

Obwohl für die Herausbildung der Muttertier-Nachkommen-Beziehung durch den Aufenthalt der Kuh-Kalb-Paare für einige Tage in den Abkalbeboxen günstige Bedingungen gegeben waren, konnte das maternale Investment, bei dem sowohl die materielle Komponente, die Milch, als auch die informationelle Wechselbeziehung zwischen Kuh und Kalb von Bedeutung ist, wahrscheinlich unter Stallhaltungsbedingungen nicht in der Qualität wie bei Weidehaltung wirksam werden. Die Milchleistung der Mutterkühe (Fleischrinder) wird für das „,normgerechte Wachstum“ der Kälber in den ersten 60 bis 120 LT für ausreichend gehalten (GOLZE, 1997; SCHOLZ et al., 2001; TEICHMANN, 2001). Im Verlaufe der Säugeperiode konnte bei allen Genotypen (Weidehaltung) eine sinkende tägliche Milchmenge beobachtet werden (SCHOLZ et al., 2001). Die Entwicklung der Milchleistung der Kuh wurde durch das Verhalten des Kalbes mitbestimmt. Die für die zu erbringende Leistung notwendige Futtermenge kann auch bei guter Futterbemessung in Gruppenhaltungen auf engem Raum nicht von allen Kühen in gleichem Maße erreicht worden sein. Die Gruppenzusammensetzung, die hier öfters geändert werden musste, und die Rangstellung der Tiere z. B. wirkten sich auf die Milchleistung der Kühe aus (HASEGAWA et al., 1997). Über die Beziehung und gegenseitige Beeinflussung der Rassenvertreter und der Familien sowie der Kühe unterschiedlichen Alters unter solchen Haltungsbedingungen ist wenig bekannt. Bei älteren Kühen wurden wegen der Euterform in einigen Fällen nur die vorderen Euterviertel von den Kälbern genutzt. Auswirkungen durch die Folgen von Euterentzündungen, die mehrfach festgestellt werden konnten, sind noch nicht ausreichend zu quantifizieren. Kleinere mittlere positive KMD der Kälber im Alter von 70 - 100 LT (Körpermasse 100 - $130 \mathrm{~kg}$ ) stehen mit solchen Ereignissen wie Abnahme der Milchleistung der Kühe, Umstellung der Ernährung auf die vermehrte Festfutteraufnahme, Enthornung und Umstallung in Verbindung (Abb. 1 und 3). In der folgenden Lebenszeit nutzten ältere Kälber intensiver die Fütterungszeiten der Kühe für die Festfutteraufnahme.

\section{Literatur}

DAY, M.L.; INAKAWA, K.; CLUTTER, A.C.; WOLFE, P.L.; ZALESKY, D. D.; NIELSEN, M. K.; KINDER, J.E.:

Suckling behaviour of calves with dams varying in milk production. J. Anim. Sci. 65 (1987), 1207-1212 
DREWEY, K.J.; BROWN, C.J.; HONEA, R.S.:

Relationships among factors associated with mothering ability in beef cattle. J. Anim. Sci. 18 (1959), 938-943

GOLZE, M.:

Extensive Rinderhaltung: Fleischrinder - Mutterkühe, Rassen, Herdenmanagement, Wirtschaftlichkeit. Verlags Union Agrar (1997)

HASEGAWA, N.; NISHIWAKI A.; SUGAWARA K.; ITO, I.:

The effects of social exchange between two groups of lactating primiparous heifers on milk production, dominance order, behaviour and adrenocorticl response. Appl. Anim. Behav. Sci. 51 (1997), 15-27

ILLMANN, G.; SPINKA, M.:

Maternal behaviour of dairy heifers and sucking of their newborn calves in group housing. Appl. Anim. Behav. Sci. 36 (1993), 91-98

KILEY-WORTHINGTON, M.; de la PLAIN, S.:

The behaviour of beef suckler cattle (Bos Taurus). Tierhaltung 14, Birkhäuser Verlag, Basel (1983)

LAZO, A.:

Social segregation and the maintenance of social stability in a feral cattle population. Anim. Behav. 48 (1994),1133-1141

Le NEINDRE, P.; MENARD, M.F.; GAREL, J.P.:

Suckling and drinking behaviour of newborn calves of beef or dairy cows. Ann. Rech. Vet. 10 (1979), 211-212

Le NEINDRE, P.:

Cow-calf relationships: the effect of management system. Current Topics in Veterinary Medicine and Animal Science 19 (1982), 24-34

LIDFORS, L.:

Mother-young behaviour in cattle. Report 33, Swedish University of Agricultural Sciences, Skara 1994

LIDFORS, L.; JENSEN, P.:

MARTIN, P: Behaviour of free-ranging beef cows and calves. Appl. Anim. Behav. Sci. 20 (1988), 237-247

The meaning of weaning. Anim. Behav. 32 (1984), 1257-1259

MENDL, M.; PAUL, E.S.:

Observation of nursing and sucking behaviour as an indicator of milk transfer and parental investment.

Anim. Behav. 37 (1989), 513-515

ODDE, K.G.; KIRACOFE, G.H.; SCHALLES, R.R.:

Suckling behaviour in range beef calves. J. Anim. Sci. 61 (1985), 307-309

OFTEDAL, O.T.:

Milk composition, milk yield and energy output at peak lactation: A comparative review. Symp. Zool.

Soc. London No. 51 (1984), 33-85

SCHOLZ, H.; KOVACS, A. Z.; STEFLER, J.; FAHR, R.-D.; LENGERKEN, G. v.:

Milchleistung und -qualität von Fleischrindkühen während der Säugeperiode. Arch. Tierz., Dummerstorf 44 (2001), 611-620

SCHOLZ, H.; MÖRCHEN, F.; SCHÄFER, S.; FAHR, R.-D.:

Zufütterung von Getreide an männliche Kälber aus der Mutterkuhhaltung während der Weideperiode. Arch. Tierz., Dummerstorf 45 (2002), 511-521

SPINKA, M.; ILLMANN, G.:

Suckling behaviour of young dairy calves with their own and alien mothers. Appl. Anim. Behav. Sci. 33 (1992), 165-173

STEINHARDT, M.; THIELSCHER, H.-H.:

Herzfrequenz und Tagesperiodik bei Saugkälbern der Mutterkuhhaltung an verschiedenen Alterspunkten. Tierärztl. Umschau 56 (2001), 206-215

STEINHARDT, M.; THIELSCHER, H.-H.:

Effekte der Entwicklungsqualität auf die Herzfrequenz, die Aktivitäts- und Ruhezeiten und deren Rhythmizität sowie auf die Wachstumsleistung der Milchrindkälber während der Aufzucht in Gruppenhaltung mit Tränkeautomatenfütterung. Arch. Tierz., Dummerstorf 45 (2002), 523-534

STEINHARDT, M.:

Effekte des Alters und der Jahreszeit auf die Aktivitäts- und Ruhezeiten sowie auf die Herzfrequenzkennwerte von Saugkälbern der Mutterkuhherde während der Weidehaltung. Tierärztl. Umschau 58 (2003), 259-268

TEICHMANN, S.:

Beziehungen zwischen Futteraufnahme, Milchmenge und Milchinhaltsstoffen, Energieretention sowie klinisch-chemischen Blutparametern bei Mutterkühen. Universität Leipzig, Diss. (2001)

VEISSIER, I.; LAMY, D.; le NEINDRE, P.: 
Social behaviour in domestic beef cattle when yearling calves are left with the cow for the next calving. Appl. Anim. Behav. Sci. 27 (1990), 193-200

VEISSIER, I.; BOISSY, A.; NOWAK, R.; ORGEUR, P.; POINDRON, P.:

Ontogeny of social awareness in the domestic herbivores. Appl. Anim. Behav. Sci. 57 (1997), 233-245

Eingegangen: 22.05.2003

Akzeptiert: 05.12.2003

Autor für Korrespondenz

Dr. habil. MARTIN STEINHARDT

Trenthorst 18

D-23847 Westerau 